\title{
Opportunities for Proxemic Interactions in Ubicomp (Keynote)
}

\author{
Saul Greenberg \\ Department of Computer Science, University of Calgary, Calgary, AB, Canada T2N 1N4 \\ saul.greenberg@ucalgary.ca
}

\begin{abstract}
In this keynote presentation, I describe and illustrate proxemic interactions as realized in several projects in my laboratory. My goal is to advocate proxemics as a more natural way of mediating inter-entity interactions in ubiquitous computing environments, while still cautioning about the many pitfalls around its use.
\end{abstract}

Keywords: Proxemic interactions, ubiquitous computing, interaction techniques, ubicomp ecologies.

\section{Introduction}

In the everyday world, much of what we do as social beings is dictated by how we interpret spatial relationships. This is called proxemics, and is part of the glue that comprises our natural social fabric. What is surprising is how little people's expectations of spatial relationships are used in interaction design within ubiquitous computing - ubicomp [1] - ecologies, i.e., in terms of mediating people's interactions with surrounding digital devices such as digital surfaces, mobile phones, tablets, computers, and other information appliances. Proxemic interaction imagines a world of devices that have fine-grained knowledge of nearby people and other devices - how they move into range, their precise distance, their identity, their orientation and their context - and how such knowledge can be exploited to design interaction techniques. Just as people expect increasing engagement and intimacy as they approach others, so should they naturally expect increasing connectivity and interaction possibilities as they bring themselves and their devices in close proximity to one another and to other things in their everyday ecology.

This keynote describes and illustrates proxemic interactions ${ }^{1}$. My goal is to advocate proxemics as a more natural way of mediating inter-entity interactions in ubicomp environments, while still cautioning about the many pitfalls around its use.

\section{What Is Proxemics?}

Anthropologist Edward Hall [7] introduced proxemics as an area of study that identifies ways that people use inter-personal distance to understand and mediate their

\footnotetext{
${ }^{1}$ The keynote address and this article is largely based upon [2], with many inspirations drawn from [3-6] as well as the myriad of wonderful works done by other researchers.
} 
interactions with other people. He was primarily concerned with culturally-dependent attributes of proxemics, where (for example) people from a Western society would consider inter-personal distance somewhat differently from people in an Arabic Society. Along the way, he developed a theory of proxemics that articulates how different things affect people's perception of inter-personal distance.

First are his definitions of four proxemic 'zones', which correlate physical to social distance. In essence, the closer the distance the more it leads to increasing expectations (and perhaps violations if the social conditions don't warrant it) of inter-personal engagement and intimacy. A second factor that influences people's proxemics interactions are the location of fixed-and semi-fixed features within the space. Fixed features include those that mark boundaries (e.g., entrances to a particular type of room), where people tend to organize certain kinds of social activities within these boundaries. Semi-fixed features are entities whose position can affect whether the space tends to bring people together, or move them apart e.g., the placement of furniture. Of course, many other factors influence proxemics. These include the social relations of the people involved, gaze and eye contact, voice volume, posture, body language, cultural taboos about touch, contextual factors such as crowding, and so on.

\section{How Can We Adapt Proxemics to Ubicomp?}

Now comes the speculation: Can we apply the theory of proxemics to ubicomp? I restate this as a working hypothesis:

Just as people expect increasing engagement and intimacy as they approach others, so should they naturally expect increasing connectivity and interaction possibilities as they bring themselves and their devices in close proximity to each other and to other things in the ecology.

The caveat is that proxemics, as envisioned by Hall and others that followed, never included ubicomp as part of its theory. Proxemics was about people's relationship to people, not to devices. Even so, I believe that proxemics can be applied as a firstorder approximation that characterizes not only people to people, but people to device and device to device inter-entity relationships and expectations. This is not as farfetched as it seems, especially because sociologists have found that people often react to computational devices as social entities, e.g., [8].

Our first challenge is to operationalize the concept of proximity in ubicomp. For devices to react, devices must be able to capture and use information from the environment as variables. While there are likely many variables we could use, we previously identified 5 variables - which we call proxemic dimensions - as a starting point [2].

- Distance between entities is fundamental. It can be captured as a continuous measure (e.g., a value between 0 - 6 feet), as a discrete measure of what zone an entity is in with respect to another entity (e.g., $[9,10])$, or even as just a binary measure, e.g., one entity is or is not in the same room as another entity.

- Orientation captures the way one entity is facing another (e.g., [11]). It too can be precise and continuous (e.g., the exact pitch/roll/yaw angle of one entity relative to another), or discrete (e.g., facing towards, somewhat towards, or away 
from the other object). Of course, orientation only makes sense if an entity has a 'front face' to it.

- Identity uniquely describes the entity. This can include exact identity and attributes, to a less detailed measure such as an entity's type, to a minimal measure that simply discriminates one entity from another.

- Movement captures the distance and orientation of an entity over time, where different actions can be taken depending on (for example) the speed of motion, and/or whether one entity is moving and turning towards vs. away from another entity.

- Location describes the physical context that the entities reside in. It can capture contextual aspects, such as when an entity crosses a threshold (a fixed feature) marking its presence or departure from a room. Crucially, the meaning applied to the four other inter-entity measures may depend on the contextual location, for example, what 'rules' get fired in different locations.

Each measure has appeared before in other ubicomp systems, and various researchers have applied one or more of these to proximity (e.g., [9-19]). Yet very few make use of all of them, let alone consider them as characterizing the interplay between entities in an ubicomp ecology. One of the hurdles is that most developers of these kinds of systems have to construct all the low-level sensing from scratch, which can significantly hinder prototype development and iteration [20]. To mitigate this problem, we developed the Proximity Toolkit $[21,6]$, a toolkit that tracks the above variables and presents them to the programmer both visually and through an API that characterizes the relationship between entities.

\section{Vignettes of What We Can Do with Proxemics}

Our efforts in prototyping software in our laboratory, as well as efforts by other researchers who have worked in the area, have produced many different examples of how knowledge of proxemics can be applied to interaction design. I characterize some of the things that have been done to illustrate the many opportunities available, via a scenario of vignettes based on the ubicomp ecology - a room - illustrated in Fig. 1. Each vignette is preceded by a heading that describes how proxemics is used to advantage. I don't go into any interface specifics here, but the references included provide pointers to particular systems that exploit and implement these strategies.

Power management and sustainability. John enters the room. Both the large display on the wall and the picture frame on the table (the information appliance) recognize that a person has crossed the threshold (the fixed feature) into the room. Each powers itself up from sleep mode so as to be readily available. When John leaves, they immediately go back to sleep. [20]

Initial state / Ambient display. Each display shows information appropriate for a justentering person who may not be directly attending the display. In this case, the wall display shows the media player John had on when he was last in the room. It highlights - using large images and minimal text appropriate for a quick glance at a distance - a few videos that he was considering watching [4]. 


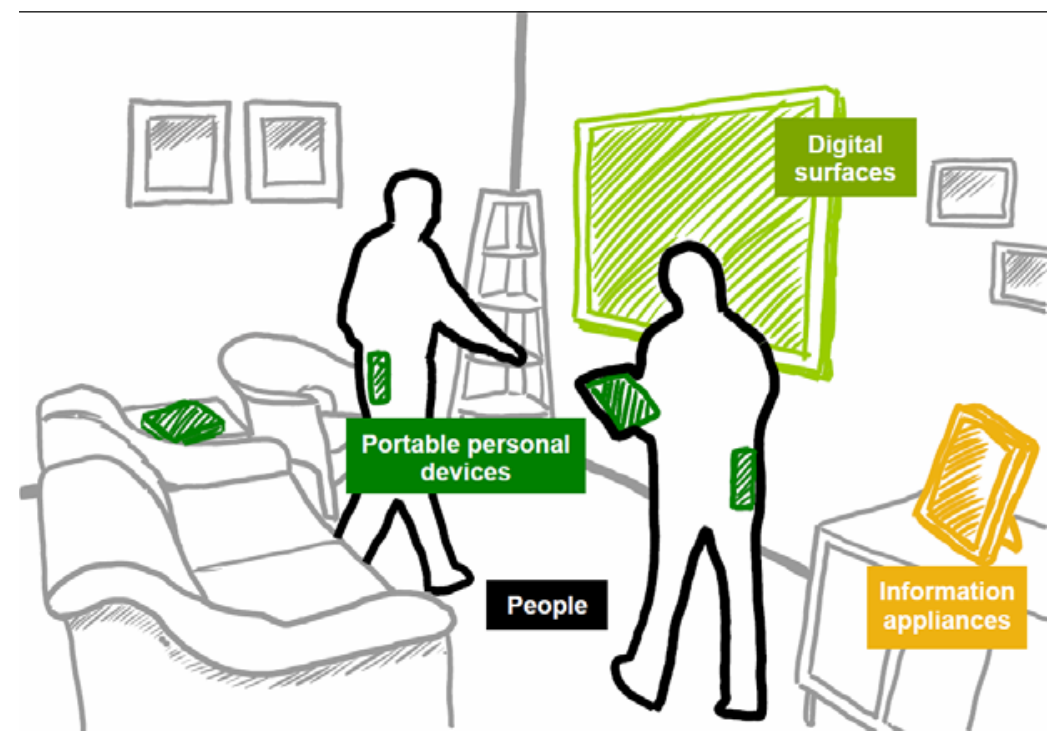

Fig. 1. Our scenario's ubicomp ecology, reproduced from [2]

Ambient to foreground interaction. The display catches John's attention, so he turns towards it and moves closer. As he does, the contents of the video player changes progressively. It shows increasingly more movies and more detail about each movie. When the display is within reach, touch controls appear so he can interact with it to choose and control videos $[2,9,10,16,4]$.

Connection possibilities. John actually wants to play a video he has on his smart phone, so he pulls it out of his pocket. As he does, he sweeps it towards the wall display, but before it gets there, it is oriented towards the picture frame. The picture frame senses that the phone is oriented towards it, and a small icon appears on the picture frame that shows the two can connect. John keeps sweeping the phone across the room. When it is facing the large display, that display illustrates a connection icon.

Establishing connections. After a few moments of orienting the phone towards the display, the icon changes to show an image of his smart phone, indicating the two are connected [14, 15, 4]. Alternately, John could have just walked up to the display, pulled his phone out of his pocket, and touched the display with it [19].

Awareness of contents. As John approaches the wall display with his phone, the position of the icon on the wall display changes its position to follow the approaching phone. At the same time, the icon expands. First, the icon shows that it (i.e., the smart phone) contains several videos. As John moves even closer, the icon smoothly displays several images around it, each identifying one of the videos on the smart phone $[2,4]$. 
Information transfer from up close. When he is within reach, John can transfer a video from his phone to the wall by several ways. He can just drag one off the icon, or he can pick and drop a video directly from his phone to the wall, or he can touch the relevant video image on the wall display with his phone $[4,3,22]$.

Information transfer from afar. Alternately, John could have used a ray-casting technique as he was approaching the wall. He touches the desired video on his phone, and points the phone to the wall display: the video appears there as an icon as if it were ray-cast. A small flick of the phone transfers it across [4, 3].

Implicit actions. John turns back, sits on the couch and faces the screen. The system recognizes this, fills the screen with the last selected video, and starts playing it $[16,4,3]$.

Attentive user interface. Part-way through the movie, John gets a phone call on his phone. He pulls it out of his pocket and presses it against his ear. The environment senses this relation of the phone to his head, and as a result the video play pauses. As soon as John puts the phone away and turns towards the screen, play resumes $[11,4,3]$.

Multiple people. While John is watching, Shelly enters. Knowing that John is still watching but that Shelly had just entered, the wall display adjusts the video slightly to show its title. As Shelly moves closer, the running video is made a bit smaller and descriptive text appears near where Shelly is standing to explain what the movie is about $[2,10,4]$.

Privacy. Shelly brings out her smart phone and, as with John, an icon appears on the display that shows it can connect. However, the contents are revealed as abstract entities. It is only when Shelly is close to the display that the actual contents are revealed in a way that is too small for John to see from afar, and where Shelly's body is shielding it.

Security. Sammy, a neighborhood kid, is visiting John's son. He knows John's system reacts to proximity, and would love to illicitly download some of John's action movies onto his smart phone, as his mom doesn't normally let him watch them. He goes into the room next door, where he stands on the other side of the wall holding the large display, brings out his phone, and points to it. However, the wall display ignores this, as the phone - even though it is close by - is oriented towards its rear.

Enhancing social security. Sammy then goes into the room and asks John if he could download some action movies. John, knowing about his mom's stance, says no but suggests he can grab some family movies. Sammy transfers one to his phone in the manner described above. Sometimes later, John is done with his movie and leaves. Sammy enters the room and tries to download an action movie. In this case, the display doesn't allow video transfers because John - who lives in the house - is not present.

The dark side \#1. John re-enters later with his lover. They have no plans to watch video, but the screen turns on showing the latest action movies as they walk by. Distracted by the display, the intimate mode is broken. 
The dark side \#2. John is watching the hockey game (one of the option he can choose). His friend, who is on the road, had asked John to phone him and give him a play by play description of the game's progress. John wants to do this, but he cannot as the play pauses whenever he brings the phone to his ear.

The dark side \#3. John has actually bought his large display at a highly reduced rate. Advertisers subsidized the cost, and John has agreed to have advertisements directed to him. Whenever John walks by, a commercial targeted to the things he usually buys appears. If he doesn't pay attention to it, the volume increases and the commercial gets busier, trying to attract his attention. It only goes away if he spends at least 10 seconds facing the ad [6].

\section{Summary}

The above examples are just a few of the opportunities (and annoyances) possible. Others exist in the current literature, and many more remain to be discovered. My hope is to stimulate your imagination, where you use these and other examples and concepts as a starting point to your own investigations.

Bio. Saul Greenberg is a Full Professor in the Department of Computer Science at the University of Calgary. He holds the NSERC/iCORE/Smart Technologies Industrial Chair in Interactive Technologies, and a University Professorship - a distinguished University of Calgary award recognizing research excellence. He received the CHCCS Achievement award in May 2007, and was also elected to the prestigious ACM CHI Academy in April 2005 for his overall contributions to the field of Human Computer Interaction.

While he is a computer scientist by training, the work by Saul Greenberg and his talented students typify the cross-discipline aspects of Human Computer Interaction, Computer Supported Cooperative Work, and Ubiquitous Computing. His many research contributions are bound by the common thread of situated interaction, which considers how computer technology fits within the fabric of people's day to day activities. This includes how such technology blends naturally in the flow of people's work practices, how people socialize and work together through technology, and how that technology fits within people's physical environment. He and his crew are well known for various significant contributions to the field, all necessary to pursue the broad goal of situated interaction:

- Articulation of design-oriented social science theories that serve as a requirements specification.

- Innovative and seminal system designs based on observations of social phenomenon.

- Toolkits enabling rapid prototyping of innovative groupware and ubiquitous appliances, and that exploit capabilities of multi-touch surfaces.

- Refinement of evaluation methods. 
Acknowledgments. This research was partially funded by the iCORE/NSERC/ SMART Technologies Industrial Research Chair in Interactive Technologies, and by the SURFNET NSERC Research Network. I am indebted to Nicolai Marquardt, Till Ballendat, Rob Diaz Marino, and Miaosen Wang: all contributed heavily to the ideas presented here.

\section{References}

1. Weiser, M.: Ubiquitous Computing. Computer 26, 71-72 (1993)

2. Greenberg, S., Marquardt, N., Ballendat, T., Diaz-Marino, R., Wang, M.: Proxe-mic interactions: the new ubicomp? Interactions 18, 42-50 (2011)

3. Ballendat, T.: Visualization of and Interaction with Digital Devices around Large Surfaces as a Function of Proximity. Diplom Thesis, University of Munich, Germany (2011)

4. Ballendat, T., Marquardt, N., Greenberg, S.: Proxemic interaction: designing for a proximity and orientation-aware environment. In: ACM International Conference on Interactive Tabletops and Surfaces, pp. 121-130. ACM Press, Saarbrucken (2010)

5. Marquardt, N.: Proxemic interactions in ubiquitous computing ecologies. In: Proceedings of the Extended Abstracts of the 2011 Annual Conference on Human Factors in Computing Systems, pp. 1033-1036. ACM Press, New York (2011)

6. Marquardt, N., Diaz-Marino, R., Boring, S., Greenberg, S.: The Proximity Tool-kit: Prototyping Proxemic Interactions in Ubiquitous Computing Ecologies. In: Proc. ACM Conference on User Interface Software and Technology, UST 2011, ACM Press, New York (2011)

7. Hall, E.T.: The Hidden Dimension. Anchor Books, New York (1963)

8. Reeves, B., Nass, C.: The Media Equation: How People Treat Computers, Tele-vision, and New Media Like Real People and Places. Cambridge University Press, Cambridge (1996)

9. Carsten, T.P., Röcker, C., Streitz, N., Stenzel, R., Magerkurth, C.: Hello.Wall - Beyond Ambient Displays. In: Adjunct Proceedings of Ubicomp, pp. 277-278 (2003)

10. Vogel, D., Balakrishnan, R.: Interactive public ambient displays: transitioning from implicit to explicit, public to personal, interaction with multiple users. In: Proceedings of the 17th Annual ACM Symposium on User Interface Software and Technology, pp. 137-146. ACM Press, USA (2004)

11. Shell, J.S., Selker, T., Vertegaal, R.: Interacting with groups of computers. Commun. ACM 46, 40-46 (2003)

12. Cooperstock, J.R., Smith, K.C.: Reactive Environments: Throwing away your keyboard and mouse. Communications of the Association of Computing Machinery (CACM) 40, 65-73 (1997)

13. Fitzmaurice, G.W.: Situated information spaces and spatially aware palmtop computers. Commun. ACM 36, 39-49 (1993)

14. Gellersen, H., Fischer, C., Guinard, D., Gostner, R., Kortuem, G., Kray, C., Rukzio, E., Streng, S.: Supporting device discovery and spontaneous interaction with spatial references. Personal Ubiquitous Comput. 13, 255-264 (2009)

15. Holmquist, L.E., Mattern, F., Schiele, B., Alahuhta, P., Beigl, M., Gellersen, H.-W.: Smart-Its Friends: A Technique for Users to Easily Establish Connections between Smart Artefacts. In: Abowd, G.D., Brumitt, B., Shafer, S. (eds.) UbiComp 2001. LNCS, vol. 2201, pp. 116-122. Springer, Heidelberg (2001) 
16. Ju, W., Lee, B.A., Klemmer, S.R.: Range: exploring implicit interaction through electronic whiteboard design. In: Proceedings of the 2008 ACM Conference on Computer Supported Cooperative Work, pp. 17-26. ACM Press, USA (2008)

17. Rekimoto, J., Ayatsuka, Y., Kohno, M., Oba, H.: Proximal Interactions: A Direct Manipulation Technique for Wireless Networking. In: Interact (2003)

18. Shoemaker, G., Tsukitani, T., Kitamura, Y., Booth, K.S.: Body-centric interaction techniques for very large wall displays. In: Proceedings of the 6th Nordic Conference on Human-Computer Interaction: Extending Boundaries, pp. 463-472. ACM Press, USA (2010)

19. Tandler, P., Prante, T., Müller-Tomfelde, C., Streitz, N., Steinmetz, R.: ConnecTables: Dynamic Coupling of Displays for the Flexible Creation of Shared Workspaces. In: Proceedings of the 14th Annual ACM Symposium on User Interface Software and Technology (UIST 2001), pp. 11-20. ACM Press, New York (2001)

20. Greenberg, S.: Toolkits and interface creativity. Multimedia Tools Appl. 32, 139-159 (2007)

21. Diaz-Marino, R., Greenberg, S.: The proximity toolkit and ViconFace: the video. In: Proceedings of the Extended Abstracts of the 28th International Conference on Human Factors in Computing Systems, pp. 4793-4798. ACM Press, USA (2010)

22. Rekimoto, J.: Pick-and-drop: a direct manipulation technique for multiple computer environments. In: Proceedings of the 10th Annual ACM Symposium on User Interface Software and Technology, pp. 31-39. ACM Press, USA (1997) 